\title{
INTERPRETIVE SUMMARIES, DECEMBER 2011
}

Dietary supplementation with nonconventional feeds from the Middle East: Assessing the effects on physicochemical and organoleptic properties of Awassi sheep milk and yogurt. By Hilali et al., page 573\%. The livelihoods of resource-poor dairy farmers in the Middle East are being affected by drought, rangeland degradation, and increasing feed costs. Poorly utilized agricultural byproducts and other nonconventional feeds could be used as low-cost supplements in sheep diets, although effects on animal production and milk quality have yet to be fully tested. Yogurt is one of the main sources of income in dairy sheep farming systems in the region, with its market price related to firmness and appearance. Treated straw, sugar beet pulp, molasses, and vetch were tested and found to affect yogurt characteristics.

Cryo-transmission electron tomography of native casein micelles from bovine milk. By Trejo et al., page 5770. Casein micelles are the primary protein component in milk and are responsible for important biological and technological properties. The aim of this study is to visualize the 3-dimensional and internal organization of the native casein micelle by cryo-electron microscopy, in the absence of the artifacts associated with traditional electron microscopy methods that require dehydration, fixation, and staining. Tomographic reconstruction showed that casein micelles contain channels and internal cavities that explain the micelle's ability to nonspecifically bind macromolecules.

Effectiveness of mid-infrared spectroscopy for the prediction of detailed protein composition and contents of protein genetic variants of individual milk of Simmental cows. By Bonfatti et al., page 5776. Mid-infrared spectroscopy was used to predict the detailed milk protein composition of a large sample of Simmental cows. Mid-infrared spectroscopy is not directly applicable when predicting detailed milk protein composition with high accuracy, as needed for the milk payment system, but the investigated calibration models might be implemented as a tool to provide breeding organizations with information on indicator traits for milk protein composition and the opportunity to enhance protein composition through indirect selection.

Lipolysis and proteolysis profiles of fresh artisanal goat cheese made with raw milk with 3 different fat contents. By Sánchez-Macías et al., page 5786. A market exists for new artisanal dairy products such as low-fat goat cheese made using traditional methods. We report the effects of milk fat reduction on proteolysis and lipolysis levels in cheese made from raw goat milk using artisanal methods. Fat reduction decreased the rate of protein degradation and the free fatty acid content in cheese, which is known to decrease cheese palatability. Thus, alternative procedures should be sought to produce healthy cheese from raw goat milk that is desirable for consumers.

Influence of calcium on $\beta$-lactoglobulin denaturation kinetics: Implications in unfolding and aggregation mechanisms. By Petit et al., page 5794. $\beta$-Lactoglobulin ( $\beta-L G$ ) is the predominant whey protein in milk products and its denaturation/aggregation (responsible for the product texture) and fouling reactions (requiring the frequent cleaning of heat exchangers) under heat treatment are mainly governed by its kinetic behavior. However, the major influence of the chemical environment on $\beta$-LG denaturation mechanisms is still not fully understood. For instance, calcium is known to promote $\beta-\mathrm{LG}$ denaturation, but this effect has rarely been quantified in terms of kinetic and thermodynamic data. This study discusses the $\beta-\mathrm{LG}$ denaturation kinetic and thermodynamic parameters, which were obtained empirically between 68 and $96^{\circ} \mathrm{C}$ for calcium concentrations up to $264 \mathrm{mg} / \mathrm{kg}$.

及-Galactosidase with transgalactosylation activity from Lactobacillus fermentum K4. By Huo et al., page 5811. The $\beta$-galactosidase from Lactobacillus fermentum K4 produces glucose and galactose by hydrolysis and prebiotic galacto-oligosaccharides (GOS) by transgalactosylation. The optimum $\mathrm{pH}$ range is relatively broad, with enzyme stability occurring around neutral $\mathrm{pH}$. The optimum temperature range for lactose utilization is 45 to $50^{\circ} \mathrm{C}$. The enzyme activity was stimulated by ethanol (15\%,wt/vol) and some cations, including $\mathrm{Na}^{+}, \mathrm{K}^{+}$, and $\mathrm{Mg}^{2+}$. Formation of GOS was rapid and dependent on lactose availability; GOS yield could reach $37 \%$ with $40 \%$ (wt/vol) lactose solution.

Modeling rennet coagulation time and curd firmness of milk. By Bittante, page 5821. The output of computerized renneting meters (e.g., Formagraph) can be interpreted using a simple model that accounts for almost all data variability and that uses only 3 parameters: rennet coagulation time, potential asymptotical curd firmness, and curd firming rate constant. The model explains the curd-firming process in milk samples varying in quality, from cows of different ages and days in milk, reared on different farms. The firming process of very late coagulating samples is not well explained by the model.

Potential for improving the carbon footprint of butter and blend products. By Flysjo, page 5833. 
To reduce greenhouse gas (GHG) emissions most efficiently, the entire life cycle of a product needs to be accounted for. Raw milk production accounts for the largest share of GHG emissions for butter. Reducing emissions at the farm level results in relatively high benefit for the final butter product. Minimizing losses throughout the product chain is important in reducing the environmental impact. Shifting to lower fat and a higher proportion of vegetable oil while retaining the functionality (e.g., taste) of butter would reduce GHG emissions.

Interactions between whey proteins and salivary proteins as related to astringency of whey protein beverages at low pH. By Ye et al., page 5842. The use of whey proteins in beverages is becoming increasingly common because of their high nutritional value and the increasing evidence of the role of whey proteins in weight management, satiety, and sarcopenia. However, whey protein beverages are known to be astringent at low $\mathrm{pH}$, which has limited consumer acceptance of these products. This study attempted to understand the mechanism of astringency by examining interactions between whey proteins and saliva in vitro for a wide range of $\mathrm{pH}$ values. This paper provides useful information on the relationship between astringency of whey protein beverage and aggregation of proteins. A sensory analysis of whey protein solution was carried out to correlate sensory and physicochemical data.

Spoilage potentials and antimicrobial resistance of Pseudomonas species isolated from cheeses. By Arslan et al., page 5851. Confidence in the safety and integrity of the food supply is an important requirement for consumers. Quality includes all attributes that influence a product's value to the consumer and can involve negative attributes such as spoilage and contamination with psychrotrophic Pseudomonas spp. Factors contributing to potential hazards in foods include poor hygiene at all stages of the food chain from producer to consumer, contaminated raw materials, and improper storage. Food control systems must address all stages of this chain. The present study emphasizes the need for food producers, public authorities, and consumers to gain awareness about the consumption of dairy products.

Identification of lactose ureide, a urea derivative of lactose, in milk and milk products. $B y$ Suyama et al., page 585\%. Due to the widespread consumption of milk, a complete characterization of its constituents and those of milk products is important in terms of functionality and safety. In this study, a novel nonreducing carbohydrate was separated from milk and powdered milk products and identified as lactose ureide. The compound might be formed by the Maillard-type reaction between lactose and urea in milk and milk products during thermal processing.

Short communication: The complete genome sequence of Bifidobacterium animalis subspecies animalis ATCC $25527^{\mathrm{T}}$ and comparative analysis of growth in milk with $B$. animalis subspecies lactis DSM 10140 $^{\mathrm{T}}$. By Loquasto et al., page 5864. The complete genome of Bifidobacterium animalis ssp. animalis (BAA) was determined and compared with that of strains of the closely related and widely used probiotic organism Bifidobacterium animalis ssp. lactis (BAL). It has been speculated that BAL has adapted to grow in milk; however, comparative analysis of both genomes did not reveal significant differences in the genes considered important for this phenotype. Furthermore, changes in bacterial count $(\mathrm{cfu} / \mathrm{mL})$ and $\mathrm{pH}$ were statistically similar between BAA and BAL after $24 \mathrm{~h}$ of growth in milk. The ability to hydrolyze milk proteins appear to limit growth of both BAA and BAL in milk.

Short communication: Technological and genotypic comparison between Streptococcus macedonicus and Streptococcus thermophilus strains from the same dairy environment. By Blaiotta et al., page 5871. Strains belonging to the species Streptococcus thermophilus and Streptococcus macedonicus coming from a premium quality production of Provolone del Monaco cheese were genetically characterized and evaluated for biochemical features of technological interest.

Associations of prepartum plasma cortisol, haptoglobin, fecal cortisol metabolites, and nonesterified fatty acids with postpartum health status in Holstein dairy cows. By Huzzey et al., page 5878. Blood and feces were sampled from 412 Holstein cows beginning 3 wk before calving. Health status was monitored for up to $30 \mathrm{~d}$ after calving. Prepartum plasma haptoglobin and plasma cortisol were not strong predictors of postpartum health status. Multiparous cows that developed more than one disorder (retained placenta, displaced abomasum, subclinical ketosis, high haptoglobin) or that died after calving had greater concentrations of fecal cortisol metabolites during wk -2 and greater concentrations of nonesterified fatty acids (NEFA) during wk $-3,-2$, and -1 , relative to calving compared with cows that did not develop these health disorders. Prepartum NEFA is a more suitable analyte than fecal cortisol metabolites to identify opportunities to improve postpartum health.

Effect of human-animal relationship and management on udder health in Swiss dairy herds. By Ivemeyer et al., page 5890. In udder health manage- 
ment, the preferred approach is to prevent, rather than treat, mastitis. Besides specific risk factors, the level of stress experienced by cows has been previously detected as a mastitis risk factor. The human-cow relationship can largely influence the level of stress of cows. The aim of this study was to investigate the effect of the human-animal relationship on udder health, and also to take management factors into account. The percentage of positive interactions of the stockperson directed towards the cows in relation to all interactions during milking had a notable effect on udder health indicators.

Serum concentration and mRNA expression in milk somatic cells of toll-like receptor 2 , toll-like receptor 4, and cytokines in dairy cows following intramammary inoculation with Escherichia coli. By Ma et al., page 5903. The aim of this study was to investigate important factors involved in innate immune responses of dairy cows to Escherichia coli-induced mastitis. Serum concentration and mRNA expression in milk somatic cells of toll-like receptor (TLR)2, TLR4, and cytokines were measured. Soluble forms of TLR2 and TLR4 may protect the host by sequestrating pathogen-associated molecular patterns during Escherichia coli mastitis.

Extended biofilm susceptibility assay for Staphylococcus aureus bovine mastitis isolates: Evidence for association between genetic makeup and biofilm susceptibility. By Melchior et al., page 5926. The antimicrobial susceptibility of Staphylococcus aureus bovine mastitis isolates growing in biofilm was assessed with an extended biofilm susceptibility (EBS) assay, with the aim of modeling therapy decisions and strain characteristics in the EBS assay. The strains were genotyped for several biofilm-related genes. The model revealed differences between genotypes, showed that better therapy results are achieved with longer duration of antimicrobial treatment, and indicated that better therapy results for penicillin-susceptible Staph. aureus isolates occurred in only a specific subgroup of strains. These insights have implications for therapeutic decisions in dairy practice.

A multi-level hierarchic Markov process with Bayesian updating for herd optimization and simulation in dairy cattle. By Demeter et al., page 5938. In this study, a herd optimization and simulation model for dairy cattle was developed. The model determines economically optimal insemination and replacement strategies for individual cows and simulates whole-herd results following from the optimal strategies. The model can be used to study various aspects of dairy farming systems, including herd management, animal health management, animal breeding, animal nutrition, and ecological sustainability. In the paper, the model was used to determine the effect of prices and production parameters on optimal replacement strategies and herd performance.

Between-cow variation in dermal fibroblast response to lipopolysaccharide reflected in resolution of inflammation during Escherichia coli mastitis. By Kandasamy et al., page 5963. Between-cow variation in innate response was studied using dermal fibroblasts as a model cell type. Early lactation cows were ranked on the basis of their fibroblast production of interleukin-8 to lipopolysaccharide treatment. Groups of low responder (LR) and high responder (HR) cows were challenged by intramammary infusion of Escherichia coli. Clearance of bacteria was similar between the groups. However, HR cows had greater mammary inflammation, as indicated by more bovine serum albumin in milk, and a delayed resolution of the inflammation, as indicated by higher milk somatic cell count and lower milk production. The innate response of the LR cows was sufficient to resolve gram-negative mastitis with less deterioration of milk quality.

Effect of heat stress during the dry period on mammary gland development. By Tao et al., page 5976. Effect of prepartum heat stress (HT) on mammary gland development was evaluated. Cows were kept in 2 groups, HT or cooling (CL), from dry off to calving. Relative to HT cows, CL cows had higher dry matter intake and body weight gain and greater mammary epithelial cell proliferation before calving and produced more milk in the next lactation. These results indicate that heat stress during the dry period compromises mammary gland development before parturition and milk yield in the next lactation.

Mechanisms regulating follicle wave patterns in the bovine estrous cycle investigated with a mathematical model. By Boer et al., page $598 \%$. A normal bovine estrous cycle contains 2 or 3 waves of follicle development, and ovulation takes place in the last wave. The reason for cycles being of the 2- or 3 -wave type is unclear. We used a mathematical model of the bovine estrous cycle to investigate possible physiological mechanisms. In the model, a number of factors influencing follicle growth rate or time point of corpus luteum regression appeared to affect the number of waves per cycle. A better understanding of follicle development could help to elucidate causes of decreased fertility in dairy cows.

Blood metabolites and hormone-based programmed breeding treatments in anovular lactating dairy cows. By El-Zarkouny et al., page 6001. More ovular than anovular cows $>100 \mathrm{~d}$ in milk (DIM) were detected in estrus after synchronization treatments, but no changes in reproduction were detected. Similar treatments were applied to ovular and anovular 
cows $\leq 100$ DIM. Anovular cows with low milk yield had more DIM at first insemination compared with anovular cows with high milk yield. Cows $\leq 100$ DIM had fewer days to first insemination and to conception, and greater pregnancy rate. Anovular cows $\leq 100$ DIM had the greatest serum total protein and globulin concentrations, and the lowest tri-iodothyronine concentrations. Regardless of stage of lactation, anovular cows had greater serum globulin than ovular cows.

A field trial on the effect of propylene glycol on milk yield and resolution of ketosis in fresh cows diagnosed with subclinical ketosis. By McArt et al., page 6011. Subclinical ketosis, a metabolic disorder that can affect more than half the cows in a herd, occurs in early lactation when dairy cows are unable to adapt to the changing energy requirements necessary for substantial milk production. The objective of this study was to determine the effect of oral propylene glycol administration on ketosis resolution and milk yield in cows diagnosed with subclinical ketosis. Cows treated with propylene glycol were more likely to resolve their subclinical ketosis and less likely to develop clinical ketosis than control cows. In addition, cows treated with propylene glycol improved milk yield during early lactation.

Effects of plane of nutrition and 2,4-thiazolidinedione on insulin responses and adipose tissue gene expression in dairy cattle during late gestation. By Schoenberg and Overton, page 6021. Responses to glucose and insulin in prepartum dairy cattle are affected by dietary energy and treatment with an insulin-sensitizing agent. Cows fed a higher plane of nutrition had a less pronounced response to a glucose challenge in terms of glucose metabolism, but a greater response in fatty acid metabolism. Differences were less pronounced in response to insulin challenge. Future evaluation of dietary treatments or metabolic intervention in dry cows should include changes in glucose and fatty acid metabolism. Both dietary intervention and insulin sensitization may be beneficial for dry cows.

Effects of transforming growth factor- $\beta$ on mammary remodeling during the dry period of dairy cows. By Casey et al., page 6036. During the dry period, stromal tissue is remodeled and epithelial cells are renewed. Treating mammary tissue collected from cows at $1 \mathrm{wk}$ dry with transforming growth factor- $\beta 1$ (TGF- 31 ) increased proliferation of epithelial and stromal cells as well as activation of stromal fibroblasts into myofibroblasts. Treatment with TGF- $\beta 1$ may be an effective way to shorten the dry period by hastening mammary remodeling, thus affecting milk production and increasing the profitability of dairy farming.
Characterization of the acute lactational response to trans-10, cis-12 conjugated linoleic acid. By Harvatine and Bauman, page 6047. Trans-10, cis-12 conjugated linoleic acid (CLA), an intermediate produced in rumen biohydrogenation, is a bioactive fatty acid that inhibits milk fat synthesis. The objective was to characterize the acute response to this CLA isomer in dairy cows. Milk fat percentage decreased progressively after $2 \mathrm{~h}$, and the initial decrease involved an equal depression of de novo synthesized and long-chain fatty acids, which was followed by a more pronounced decrease in the synthesis of de novo fatty acids. The mammary gland is acutely sensitive to CLA and our results provide insight into the primary and secondary mechanisms of milk fat depression.

Prediction of enteric methane output from milk fatty acid concentrations and rumen fermentation parameters in dairy cows fed sunflower, flax, or canola seeds. By Mohammed et al., page 605\%. Methane emissions from ruminants contribute to global warming, but on-farm estimation of methane requires expensive equipment. Thus, the objectives were to develop mathematical equations that model methane emissions from milk fatty acid composition of cows fed various diets and to validate previously reported methane prediction equations using an independent dataset. Predictive equations from milk fatty acids provided a better estimate of methane emissions when developed within diets than when predicted across diets. Predictive equations from previous studies overestimated methane emissions in this study. The scope for developing universal methane prediction equations from milk fatty acid composition alone appears to be limited.

Effects of forage type, forage to concentrate ratio, and crushed linseed supplementation on milk fatty acid profile in lactating dairy cows. $B y$ Sterk et al., page 6078. Characteristics of the basal diet profoundly affect ruminal metabolism of dietary fatty acids, and consequently milk fatty acid profiles. The combined effects of crushed linseed supplementation and variation in forage type (grass versus corn silage) and forage to concentrate ratio on milk fatty acid profile were evaluated in a multivariate design experiment. The effect of adding crushed linseed on the proportions of several fatty acids in milk fat, including beneficial fatty acids for human health, depends on forage type and forage to concentrate ratio in the basal diet.

Dry and moist heating-induced changes of protein molecular structure, protein subfraction, and nutrient profiles in soybeans. By Samadi and $Y u$, page 6092. This study aimed to investigate the heat-induced effect of soybean on protein molecular 
structure and protein nutrient supply in dairy cattle. The sensitivity of soybean seeds to moist heating was much higher than the sensitivity to dry heating in terms of the structure and nutrient profile changes.

Technical note: Validation of methodology for characterization of feeding behavior in dairy calves. By Miller-Cushon and DeVries, page 6103. The objective of this study was to evaluate time-sampling methodology for the collection of feeding behavior of dairy calves. Pre- and postweaning calf feeding behavior data obtained using instantaneous recording at a range of time intervals were compared with data obtained from continuous recording. In both time periods, feeding time data obtained using instantaneous recording at 5-min intervals provided a fair representation of continuous data, but the strong association of meal frequency and meal time required instantaneous recording intervals no greater than $1 \mathrm{~min}$ and $30 \mathrm{~s}$, respectively. These results indicate that instantaneous recording can provide accurate calf feeding behavior data if the scanning interval is sufficiently short.

Technical note: Comparison of 3 methods for analyzing areas under the curve for glucose and nonesterified fatty acids concentrations following epinephrine challenge in dairy cows. By Cardoso et al., page 6111. We evaluated methods to determine responses of dairy cows to epinephrine challenge as affected by dietary or abomasally infused niacin. The response was measured by calculating area under the curve (AUC) for concentrations of nonesterified fatty acids and glucose in plasma in blood samples before and after epinephrine injection. The positive incremental method and the total area method resulted in similar results and interpretation compared with the "gold standard" incremental area method. Methods of calculating AUC may give different results.

Hot topic: Performance of bovine high-density genotyping platforms in Holsteins and Jerseys. By Rincon et al., page 6116. The performance of the Illumina High-Density Bovine BeadChip Array [777,962 single nucleotide polymorphisms (SNP)] and the Affymetrix Axiom Genome-Wide BOS 1 Array (648,874 SNP) was evaluated using DNA samples derived from Holstein and Jersey cattle. Data were edited to remove SNP having a call rate less than $90 \%$, and those in linkage disequilibrium $\left(\mathrm{r}^{2} \geq 0.9\right)$. Nineteen percent more Affymetrix SNP remained following these edits. The Illumina platform provided superior data for copy number variant analyses due to larger number of SNP, higher intensity signals, and lower background. The combined use of both platforms significantly improved coverage over either platform alone.
Genetic parameters for predicted methane production and potential for reducing enteric emissions through genomic selection. By de Haas et al., page 6122. The objective of this study was to evaluate the phenotypic variance of predicted methane emission (PME) in dairy heifers, how it is associated with milk yield and residual feed intake (RFI), and its genetic basis. Estimated heritabilities for PME and RFI were 0.35 and 0.40 . Positive genetic correlation between RFI and PME indicated that cows with lower RFI also have lower PME. Hence, it is possible to decrease methane production by selecting more efficient cows, and the genetic variation suggests that reductions of 11 to $26 \%$ in $10 \mathrm{yr}$ are theoretically possible, and in a genomic selection program even higher.

Evaluations for service-sire conception rate for heifer and cow inseminations with conventional and sexed semen. By Norman et al., page 6135 . Evaluations for Holstein and Jersey service-sire conception rate (SCR) were calculated based on cow or heifer inseminations with conventional or sexed semen. Little relationship was found between conventional and sexed semen SCR based on either cow or heifer inseminations. However, cow and heifer SCR were highly related when SCR was based on either conventional or sexed semen. Service-sire conception rates appeared to be more accurate across time when Holstein cow and heifer inseminations were combined; however, small sample sizes warrant further study in Jerseys. Separate SCR evaluations are needed for conventional versus sexed semen.

Controlling inbreeding and maximizing genetic gain using semi-definite programming with pedigree-based and genomic relationships. By Schierenbeck et al., page 6143. The tool of genomic selection will enable achieving higher genetic gain due to a substantial reduction in generation intervals. Intensive selection is associated with the risk of a further increase in genetic relationships within the German Holstein population. We evaluated an approach to consider aspects of maximizing genetic gain and controlling inbreeding in a long-term perspective based on genomic and additive genetic relationships.

Harmful recessive effects on fertility detected by absence of homozygous haplotypes. By VanRaden et al., page 6153. Based on genomic testing, a method was developed to discover lethal defects by detecting the absence of homozygous haplotypes. Five new lethal recessive defects were discovered: 3 in Holsteins, 1 in Jerseys, and 1 in Brown Swiss. Haplotype testing revealed harmful effects on sire conception rate for these 5 new defects as well as 2 previously known defects 
that were consistent with lethal recessive inheritance. Once animals have been genotyped, dairy farmers could avoid mating carrier animals without further testing expense using the new haplotype test, thereby saving time, increasing profitability, and reducing those defects in the population.

Genetic analysis of fertility in the Italian Brown Swiss population using different models and trait definitions. By Tiezzi et al., page 6162. Genetic parameters for fertility and production traits were estimated in the Italian Brown Swiss population reared in the Bolzano-Bozen province. An efficient artificial insemination recording scheme has been running in this Alpine area. Censored threshold and linear models were applied on female fertility traits, expressed both as days and cycles measured. Estimates of heritability ranged from 0.03 to 0.07 . Several fertility indicators could be exploited to cope with the deterioration of fertility in the Italian Brown Swiss breed.

Short communication: Do Holstein lactations of varied lengths have different characteristics? $B y$ Pollott, page 6173. Lactation lengths of Holstein cows have been increasing in recent years. When using data for modeling dairy cow production, it is important to know if the characteristics of a lactation depend on its length. Data from over 29,000 lactations, divided in 305-d, 370-d, and 440-d lactation length categories, were analyzed. All aspects of the lactations (daily milk production, fat, protein, and lactose contents of the milk) differed depending on the length of the lactation. In addition, pregnancy altered these features of milk production such that the usual changes in late lactation were more rapid once pregnancy reached the fourth month.

Short communication: Genetic parameters for measures of calf health in a population of Holstein calves in New York State. By Henderson et al., page 6181. Calf health is a major concern for dairy producers. Calfhood disease can have severe economic consequences and long-term effects on the profitability of an animal. Genetic variation exists among sires for 3 analyzed calf health traits: bovine respiratory disease, bloat, and navel infections. The heritabilities of the 3 health traits ranged from 0.04 to 0.14 . The genetic correlation between respiratory disease and bloat was 0.62 , whereas it was close to zero among other health trait pairs. Significant associations exist among estimated breeding values for calf health traits and other routinely evaluated traits in Canada and the United States.

Technical note: Adjustment of traditional cow evaluations to improve accuracy of genomic predictions. By Wiggans et al., page 6188. A method of adjusting the genetic evaluations of genotyped cows was developed that increased the accuracy of genomic evaluations. Cow evaluations were adjusted to have characteristics similar to those of bull evaluations. The adjustment used the same mean and variance adjustment for all genotyped cows in a breed. The adjustment method was implemented in April 2010 and used until April 2011, when it was revised and expanded to include all cows. Genomic evaluations became more accurate because information from cows was used more effectively.

Solubility of commercial milk protein concentrates and milk protein isolates. By Sikand et al., page 6194. Our study focused on determining the solubility of commercially available milk protein concentrate (MPC) and milk protein isolate (MPI) powders in the market. We observed low solubility (23 to $42 \%$ ) in MPC80 (MPC with $80 \%$ protein) samples and a wide range of solubility (33 to $98 \%$ ) in MPI samples. We observed an association between high solubility of MPI samples with greater contents of monovalent salts such as $\mathrm{NaCl}$ or $\mathrm{KCl}$ and lower contents of calcium. Our study demonstrated that mineral composition plays an important role in the solubility of high protein powders such as MPC80 and MPI.

Randomized clinical trial to evaluate the efficacy of a 5-day ceftiofur hydrochloride intramammary treatment on nonsevere gram-negative clinical mastitis. By Schukken et al., page 6203. This study evaluated the efficacy of a 5-d treatment with intramammary ceftiofur hydrochloride on clinical and bacteriological cure of gram-negative, nonsevere clinical mastitis. Treatment was compared with a nontreated control group. One hundred four cows on 5 farms were enrolled in the study. Treatment significantly improved bacteriological cure, particularly for animals infected with Escherichia coli or Klebsiella spp., and was associated with improved clinical performance of the treated cows. Treatment did not significantly affect milk production or linear score.

An economic decision-making support system for selection of reproductive management programs on dairy farms. By Giordano et al., page 6216. Reproductive management is a major contributor to dairy farm profitability. However, selection of best performing programs is difficult and complex. A highly adaptable decision support tool was created to evaluate reproductive performance and estimate the economic outcome of different reproductive management programs that use estrous detection, timed artificial insemination, or a combination of both. This decision support system is a valuable tool that may be used to assist dairy producers and industry consultants at 
the time of selecting the best reproductive management strategy to apply at a farm.

The effect of feeding dairy heifers diets with and without supplemental phosphorus on growth, reproductive efficiency, health, and lactation performance. By Bjelland et al., page 6233. Dairy heifers were fed diets with or without excess phosphorus (P) from 4 to 22 mo of age. Heifers were evaluated for body weight, external frame size, dystocia, reproductive efficiency, and first-lactation performance. Heifers offered diets with or without excess $\mathrm{P}$ performed similarly for every trait examined. The amount of $\mathrm{P}$ excretion was also examined and indicated that all excess $\mathrm{P}$ consumed by heifers was excreted in the feces and not retained. These results suggest no benefit of feeding $\mathrm{P}$ above recommended concentrations to dairy heifers.
Consequence of alternative standards for bulk tank somatic cell count of dairy herds in the United States. By Norman et al., page 6243. Consideration of changes for US standards for bulk-tank somatic cell count (BTSCC) are underway because of a European Union (EU) announcement that its standards will be enforced on any herds supplying EU imports. For herds participating in Dairy Herd Improvement (DHI) testing or shipping milk to 4 Federal Milk Marketing Orders (FMO), noncompliance was 0.9 and $1.0 \%$, respectively, for a US standard of 750,000 cells/ $\mathrm{mL}$ and 7.8 and $16.1 \%$ for a EU standard of 400,000 cells/mL. Changes in US BTSCC standards proposed without improved herd management would increase noncompliance in DHI and FMO herds up to 14.1 and $23.3 \%$, respectively. 